\title{
Nonlinear superhorizon perturbations in Hor̆ava-Lifshitz gravity
}

\author{
Keisuke Izum* \\ Yukawa Institute for Theoretical Physics, Kyoto University, Kyoto 606-8502, Japan \\ Shinji Mukohyamađ \\ IPMU, The University of Tokyo, Kashiwa, Chiba 277-8582, Japan
}

(Dated: August 6, 2018)

\begin{abstract}
We perform a fully nonlinear analysis of superhorizon perturbation in Hořava-Lifshitz gravity, based on the gradient expansion method. We present a concrete expression for the solution of gravity equations up to the second order in the gradient expansion, and prove that the solution can be extended to any order. The result provides yet another example for analogue of the Vainshtein effect: the nonlinear solution is regular in the limit $\lambda \rightarrow 1$ and recovers general relativity coupled to dark matter at low energy. Finally, we propose a definition of nonlinear curvature perturbation $\mathcal{R}$ in Hořava-Lifshitz gravity and show that it is conserved up to the first order in the gradient expansion.
\end{abstract}

\section{INTRODUCTION}

The new theory of gravitation proposed recently by Hořava [1, 2] is expected to be renormalizable and unitary. For this reason, it has been attracting significant amount of attention $[3-6]$. The theory is power-counting renormalizable because of the anisotropic scaling in the ultraviolet (UV),

$$
t \rightarrow b^{z} t, \quad \vec{x} \rightarrow b \vec{x},
$$

with the dynamical critical exponent $z \geq 3$. Since this scaling is called Lifshitz scaling, the theory is often called Hořava-Lifshitz gravity.

Because of the anisotropic scaling (10), the time and the space in this theory must be treated separately. Thus, we must abandon the 4-dimensional diffeomorphism invariance. Instead, the fundamental symmetry of the theory is the invariance under the so-called foliation-preserving diffeomorphism:

$$
t \rightarrow t^{\prime}(t), \quad \vec{x} \rightarrow \vec{x}^{\prime}(t, \vec{x}),
$$

which preserves the way the spacetime is foliated by constant-time hypersurfaces. Since this symmetry is smaller than the counterpart of general relativity i.e. the 4-dimensional diffeomorphism invariance, the structure of the action is less restrictive and allows more parameters. For example, the kinetic part of the action is a linear combination of $K^{2}$ and $K^{i j} K_{i j}$ with arbitrary coefficients, where $K_{i j}$ is the extrinsic curvature of the constant-time hypersurface and $K=K_{i}^{i}$. Hence, the kinetic part can be written as

$$
I_{k i n}=\frac{M_{P l}^{2}}{2} \int N d t \sqrt{g} d^{3} \vec{x}\left(K^{i j} K_{i j}-\lambda K^{2}\right),
$$

where $M_{P l}$ is the reduced Planck mass and $\lambda$ is an arbitrary parameter. While in general relativity the value of $\lambda$ is fixed to unity due to the 4-dimensional diffeomorphism invariance, in Horava-Lifshitz gravity any value of $\lambda$ is consistent with the foliation-preserving diffeomorphism invariance.

In Hořava-Lifshitz gravity, in addition to the usual tensor gravitons, there is an extra physical degree of freedom called the scalar graviton [1]. In order for the scalar graviton not to be a ghost, the regime $1 / 3<\lambda<1$ should be excluded [3]. Outside this forbidden interval, the scalar graviton has negative sound speed squared. Therefore, in order for the theory to be observationally viable, we need to impose the condition under which the associated long-distance instability does not show up [2]. The condition essentially says that $\lambda$ must be sufficiently close to 1 in the infrared (IR), and should be considered as a phenomenological constraint on properties of the renormalization group (RG) flow. Since the value of $\lambda$ continuously changes by the RG flow, only the regime $\lambda>1$ is allowed.

\footnotetext{
*Electronic address: izumi@yukawa.kyoto-u.ac.jp
}

${ }^{\dagger}$ Electronic address: shinji.mukohyama@ipmu.jp 
When $\lambda$ is very close to 1 , the scalar graviton gets strongly coupled [4]. If we adopt the usual metric perturbation method then we find that higher order terms in the time kinetic part of the action for the scalar graviton become larger. This indicates the breakdown of the perturbative expansion in the scalar graviton sector. Note that this does not necessarily imply the loss of predictability since all coefficients of infinite number of terms can be written in terms of finite parameters in the action if the theory is renormalizable. However, because of the breakdown of the perturbative expansion, we need to employ a more or less non-perturbative method to analyze the fate of the scalar graviton in the limit $\lambda \rightarrow 1$. Such an analysis was performed in [2] for spherically symmetric, static, vacuum configurations and it was shown that the limit is continuous and recovers general relativity. This may be considered as an analogue of the Vainshtein effect [9].

The main purpose of this paper is to provide yet another example of the analogue of the Vainshtein effect in HořavaLifshitz gravity. For this purpose, we perform a fully nonlinear analysis of superhorizon cosmological perturbation, adopting the so-called gradient expansion method [7]. The result is obviously continuous in the limit $\lambda \rightarrow 1$ and recovers general relativity coupled to dark matter.

We also propose a definition of nonlinear curvature perturbation $\mathcal{R}$ in Hořava-Lifshitz gravity and show that it is conserved up to the first order in the gradient expansion.

The paper is organized as follows. In $\S$, we briefly review the basic equations in Hořava-Lifshitz gravity. In $\S$ III. we introduce the gradient expansion method in this theory and present the solution to equations of motion. In $\S$ IV, we proposed a definition of nonlinear curvature perturbation $\mathcal{R}$ and show that it is conserved up to the first order in the gradient expansion. $\S \mathbb{V}$ is devoted to a summary of this paper. In appendix $\mathrm{A}$ we prove that the solution obtained in $\S$ III satisfies the momentum constraint in any order of the gradient expansion.

\section{BASIC EQUATIONS}

In this section we review the basic equations of Hořava-Lifshitz gravity, following the notation in [2], and reformulate them in a way suitable for gradient expansion. Basic quantities of Hořava-Lifshitz gravity are the lapse $N(t)$, the shift $N^{i}(t, \vec{x})$ and the 3 -dimensional spatial metric $g_{i j}(t, \vec{x})$. Combining these quantities, we can construct 4-dimensional spacetime metric in the ADM form as

$$
d s^{2}=-N^{2} d t^{2}+g_{i j}\left(d x^{i}+N^{i} d t\right)\left(d x^{j}+N^{j} d t\right) .
$$

The fundamental symmetry of the theory is the invariance under the so called foliation preserving diffeomorphism:

$$
t \rightarrow t^{\prime}(t), \quad \vec{x} \rightarrow \vec{x}^{\prime}(t, \vec{x}),
$$

which preserves the way the spacetime is foliated by constant-time hypersurfaces.

By requiring invariance under spatial parity and time reflection, the gravitational action is specified as

$$
I_{g}=\frac{M_{P l}^{2}}{2} \int N d t \sqrt{g} d^{3} \vec{x}\left(K_{i j} K^{i j}-\lambda K^{2}-2 \Lambda+R+L_{z>1}\right),
$$

where $\sqrt{g}$ is the determinant of $g_{i j}, K_{i j}$ is the extrinsic curvature defined as

$$
K_{i j}=\frac{1}{2 N}\left(\partial_{t} g_{i j}-D_{i} N_{j}-D_{j} N_{i}\right)
$$

$K\left(=g^{i j} K_{i j}\right)$ is the trace of $K_{i j}, D_{i}$ is the spatial covariant derivative compatible with $g_{i j}$, and $R$ is the Ricci scalar constructed from $g_{i j}$. To lower and raise an index, $g_{i j}$ and its inverse $g^{i j}$ are used. We impose the so called projectability condition, namely we require that the lapse function should depend only on time. In order to realize the power-counting renormalizability, the higher curvature Lagrangian $L_{z>1}$ should include up to sixth or higher spatial derivatives. For our analysis in this paper, we do not need to specify the concrete form of $L_{z>1}$. Moreover, for simplicity we shall not include matter action and analyze the pure gravity described by the action (6).

The equation of motion for $g_{i j}$ is $\mathcal{E}_{g i j}=0$, where

$$
\begin{aligned}
\mathcal{E}_{g i j} \equiv & g_{i k} g_{j l} \frac{2}{N \sqrt{g}} \frac{\delta I_{g}}{\delta g_{k l}} \\
= & M_{P l}^{2}\left[-\frac{1}{N}\left(\partial_{t}-N^{k} D_{k}\right) p_{i j}+\frac{1}{N}\left(p_{i k} D_{j} N^{k}+p_{j k} D_{i} N^{k}\right)\right. \\
& \left.-K p_{i j}+2 K_{i}^{k} p_{k j}+\frac{1}{2} g_{i j} K^{k l} p_{k l}-\Lambda g_{i j}-G_{i j}\right]+\mathcal{E}_{z>1 i j}
\end{aligned}
$$


Here, $p_{i j} \equiv K_{i j}-\lambda K g_{i j}, \mathcal{E}_{z>1 i j}$ is the contribution from $L_{z>1}$ and $G_{i j}$ is Einstein tensor of $g_{i j}$. The trace part and traceless part of this equation are, respectively,

$$
(3 \lambda-1)\left(\partial_{\perp} K+\frac{1}{2} K^{2}\right)+\frac{3}{2} A_{j}^{i} A_{i}^{j}+Z=0,
$$

and

$$
\partial_{\perp} A^{i}{ }_{j}+K A^{i}{ }_{j}+\frac{1}{N}\left(A^{k}{ }_{j} \partial_{k} N^{i}-A_{k}^{i} \partial_{j} N^{k}\right)-\left(Z^{i}{ }_{j}-\frac{1}{3} Z \delta_{j}^{i}\right)=0
$$

where

$$
A_{j}^{i} \equiv K_{j}^{i}-\frac{1}{3} K \delta_{j}^{i}
$$

is the traceless part of $K_{j}^{i}$,

$$
\partial_{\perp} \equiv \frac{1}{N}\left(\partial_{t}-N^{k} \partial_{k}\right)
$$

and

$$
Z_{j}^{i} \equiv-\Lambda \delta_{j}^{i}-G_{j}^{i}+M_{P l}^{-2} g^{i k} \mathcal{E}_{z>1 k j}, \quad Z=Z_{i}^{i}=-3 \Lambda+\frac{1}{2} R+M_{P l}^{-2} g^{i j} \mathcal{E}_{z>1 i j}
$$

The foliation preserving diffeomorphism includes the three dimensional spatial diffeomorphism as a part of it. As a result, $Z^{i}{ }_{j}$ satisfies the generalized Bianchi identity,

$$
D_{j} Z_{i}^{j}=0 \text {. }
$$

For convenience, we decompose the spatial metric and the extrinsic curvature as

$$
\begin{aligned}
g_{i j} & =a^{2}(t) e^{2 \zeta(t, \vec{x})} \gamma_{i j}(t, \vec{x}), \\
K_{j}^{i} & =\frac{1}{3} K(t, \vec{x}) \delta^{i}{ }_{j}+A^{i}{ }_{j}(t, \vec{x}),
\end{aligned}
$$

where we define $\zeta(t, \vec{x})$ so that $\operatorname{det} \gamma=1$, and $a(t)$ is defined in eq.(30) later. The trace part and the traceless part of the definition of the extrinsic curvature lead, respectively, to

$$
\partial_{\perp} \zeta+\frac{\partial_{t} a}{N a}=\frac{1}{3}\left(K+\partial_{i} N^{i}\right)
$$

and

$$
\partial_{\perp} \gamma_{i j}=2 \gamma_{i k} A_{j}^{k}+\frac{1}{N}\left(\gamma_{j k} \partial_{i} N^{k}+\gamma_{i k} \partial_{j} N^{k}-\frac{2}{3} \gamma_{i j} \partial_{k} N^{k}\right)
$$

The momentum constraint, i.e. the equation of motion for $N^{i}$, is

$$
D_{j} K_{i}^{j}-\lambda \partial_{i} K=0 .
$$

According to the decomposition (16), the momentum constraint is rewritten as

$$
\partial_{j} A_{i}^{j}+3 A_{i}^{j} \partial_{j} \zeta-\frac{1}{2} A^{j}{ }_{l} \gamma^{l k} \partial_{i} \gamma_{j k}-\frac{1}{3}(3 \lambda-1) \partial_{i} K=0 .
$$

As a consistency check, it is instructive to calculate time derivatives of $A_{i}^{i}, \ln \operatorname{det} \gamma, \gamma_{i j}-\gamma_{j i}$ and $\gamma_{i k} A^{k}{ }_{j}-\gamma_{j k} A_{i}^{k}$ without using the fact that they actually vanish. The results are

$$
\begin{aligned}
& \partial_{\perp} A_{i}^{i}=-K A_{i}^{i}, \\
& \partial_{\perp}(\ln \operatorname{det} \gamma)=2 A^{i}{ }_{i}, \\
& \partial_{\perp}\left(\gamma_{i j}-\gamma_{j i}\right)=2\left(\gamma_{i k} A_{j}^{k}-\gamma_{j k} A_{i}^{k}\right) \text {, } \\
& \partial_{\perp}\left(\gamma_{i k} A^{k}{ }_{j}-\gamma_{j k} A_{i}^{k}\right)=-K\left(\gamma_{i k} A_{j}^{k}-\gamma_{j k} A_{i}^{k}\right)+2\left(\gamma_{i l} A_{k}^{l}-\gamma_{k l} A_{i}^{l}\right) A_{j}^{k}-2\left(\gamma_{j l} A_{k}^{l}-\gamma_{k l} A^{l}{ }_{j}\right) A^{k}{ }_{i} \\
& +2\left(\gamma_{k l}-\gamma_{l k}\right) A_{i}^{k} A_{j}^{l}+\frac{1}{N}\left(\gamma_{i k} A_{l}^{k}-\gamma_{l k} A_{i}^{k}\right) \partial_{j} N^{l}-\frac{1}{N}\left(\gamma_{j k} A_{l}^{k}-\gamma_{l k} A_{j}^{k}\right) \partial_{i} N^{l} \\
& -\frac{2}{3}\left(\gamma_{i k} A_{j}^{k}-\gamma_{j k} A_{i}^{k}\right) \partial_{l} N^{l}-\frac{1}{3} Z\left(\gamma_{i j}-\gamma_{j i}\right) .
\end{aligned}
$$

The right hand side of each equation vanishes when $A_{i}^{i}$, ln $\operatorname{det} \gamma, \gamma_{i j}-\gamma_{j i}$ and $\gamma_{i k} A_{j}^{k}-\gamma_{j k} A_{i}^{k}$ vanish. Therefore, the evolution equations we have derived are consistent with vanishing $A_{i}^{i}$, ln $\operatorname{det} \gamma, \gamma_{i j}-\gamma_{j i}$ and $\gamma_{i k} A^{k}{ }_{j}-\gamma_{j k} A^{k}$. 


\section{GRADIENT EXPANSION}

The gradient expansion is the method to analyze the full non-linear dynamics at large scale. In the gradient expansion, we consider nonlinear perturbation around a flat Friedmann-Robertson-Walker background and suppose that the characteristic spatial scale $L$ of the perturbation is much larger than the Hubble horizon scale $1 / H$. To make the argument transparent, we introduce a small parameter $\epsilon$ defined by $\epsilon \sim 1 /(H L)$ and expand all relevant quantities and equations with respect to $\epsilon$. For example, a spatial derivative acted on a relevant quantity raises the order of $\epsilon$ and thus is counted as $O(\epsilon)$. We then solve the equations order by order in gradient expansion.

\section{A. Gauge fixing}

The foliation preserving diffeomorphism invariance is, like all other gauge symmetries, redundancy of descriptions. In order to extract physical quantities and statements, we thus need to eliminate gauge freedom by imposing appropriate gauge condition. In this paper we adopt the synchronous gauge, or the Gaussian normal coordinate system, by setting the lapse to unity and the shift to zero.

$$
N=1, N^{i}=0 \text {. }
$$

This fixes the time coordinates but does not completely fix the spatial coordinates. There still remains gauge freedom of time-independent spatial diffeomorphism, corresponding to the change of coordinates on the initial constant-time hypersurface. This residual gauge degree of freedom will be discussed later.

In this gauge our basic equations (9), (10), (17) and (18) are simplified as

$$
\begin{aligned}
(3 \lambda-1) \partial_{t} K & =-\frac{1}{2}(3 \lambda-1) K^{2}-\frac{3}{2} A^{i}{ }_{j} A^{j}{ }_{i}-Z \\
\partial_{t} A_{j}^{i} & =-K A^{i}{ }_{j}+Z^{i}{ }_{j}-\frac{1}{3} Z \delta_{j}^{i}, \\
\partial_{t} \zeta & =-\frac{\partial_{t} a}{a}+\frac{1}{3} K \\
\partial_{t} \gamma_{i j} & =2 \gamma_{i k} A_{j}^{k} .
\end{aligned}
$$

Hereafter we assume that $\lambda \neq 1 / 3$. Actually, as already explained in the introduction, the regime of physical interest is $\lambda>1$.

\section{B. Order analysis}

In order to expand the equations and to write down equations in each order of gradient expansion, we need to know the orders of all relevant variables. Therefore, we begin with the order analysis to determine them.

Since we are interested in the spacetime which is not so much different from the exact Friedmann universe, we suppose that

$$
\partial_{t} \gamma_{i j}=O(\epsilon)
$$

Substituting this into eq.(26), we obtain

$$
A^{i}{ }_{j}=O(\epsilon)
$$

Then, the constraint equation (20) implies that

$$
\partial_{i} K=O\left(\epsilon^{2}\right)
$$

In other words, $K^{(0)}$ depends on $t$ only. This fact enables us to define $a(t)$ by

$$
3 \frac{\partial_{t} a(t)}{a(t)}=K^{(0)}(\equiv 3 H(t)) .
$$

With this definition of $a(t)$, eq. (25) leads to

$$
\partial_{t} \zeta=O(\epsilon)
$$


In summary we have the following expansion.

$$
\begin{aligned}
\zeta & =\zeta^{(0)}(\vec{x})+\epsilon \zeta^{(1)}(t, \vec{x})+\epsilon^{2} \zeta^{(2)}(t, \vec{x})+\cdots, \\
\gamma_{i j} & =f_{i j}(\vec{x})+\epsilon \gamma_{i j}^{(1)}(t, \vec{x})+\epsilon^{2} \gamma_{i j}^{(2)}(t, \vec{x})+\cdots, \\
K & =3 H(t)+\epsilon K^{(1)}(t, \vec{x})+\epsilon^{2} K^{(2)}(t, \vec{x})+\cdots, \\
A_{j}^{i} & =\epsilon A_{j}^{(1) i}(t, \vec{x})+\epsilon^{2} A_{j}^{(2) i}(t, \vec{x})+\cdots,
\end{aligned}
$$

where a quantity with the upper index $(n)$ is $n$-th order in gradient expansion.

\section{Equations in each order}

We have found the orders of all physical quantities. Substituting this into the evolution equations (23, 26), we can obtain the evolution equations in each order.

In the zero-th order of gradient expansion we have

$$
(3 \lambda-1)\left(\partial_{t} H+\frac{3}{2} H^{2}\right)=\Lambda
$$

The first integral of this equation leads to

$$
3 H^{2}=\frac{2 \Lambda}{3 \lambda-1}+\frac{\tilde{C}}{a^{3}}
$$

where $\tilde{C}$ is an integration constant. The second term in the right hand side of this equation is the "dark matter as an integration constant" [5].

The $n$-th $(n \geq 1)$ order equations are written as

$$
\begin{aligned}
a^{-3} \partial_{t}\left(a^{3} K^{(n)}\right) & =-\frac{1}{2} \sum_{p=1}^{n-1} K^{(p)} K^{(n-p)}-\frac{3}{2(3 \lambda-1)} \sum_{p=1}^{n-1} A_{j}^{(p) i} A_{j}^{(n-p) j}-\frac{Z^{(n)}}{3 \lambda-1}, \\
a^{-3} \partial_{t}\left(a^{3} A_{j}^{(n) i}\right) & =-\sum_{p=1}^{n-1} K^{(p)} A_{j}^{(n-p) i}+Z_{j}^{(n) i}-\frac{1}{3} Z^{(n)} \delta_{j}^{i}, \\
\partial_{t} \zeta^{(n)} & =\frac{1}{3} K^{(n)}, \\
\partial_{t} \gamma_{i j}^{(n)} & =2 \sum_{p=0}^{n-1} \gamma_{i k}^{(p)} A_{j}^{(n-p) k},
\end{aligned}
$$

In a similar way, from eq.(20) we obtain the $(n+1)$-th $(n \geq 1)$ order momentum constraint equation as

$$
\partial_{j} A_{i}^{(n) j}+3 \sum_{p=1}^{n} A_{i}^{(p) j} \partial_{j} \zeta^{(n-p)}-\frac{1}{2} \sum_{p=1}^{n} \sum_{q=0}^{n-p} A^{(p) j}{ }_{l}\left(\gamma^{-1}\right)^{(q) l k} \partial_{i} \gamma_{j k}^{(n-p-q)}-\frac{1}{3}(3 \lambda-1) \partial_{i} K^{(n)}=0
$$

where $\left(\gamma^{-1}\right)^{(n) i j}$ is the $n$-th order part of the inverse of $\gamma_{i j}$, i.e. the inverse $\left(\gamma^{-1}\right)^{i j}$ is expanded as

$$
\left(\gamma^{-1}\right)^{i j}=f^{i j}+\epsilon\left(\gamma^{-1}\right)^{(1) i j}+\epsilon^{2}\left(\gamma^{-1}\right)^{(2) i j}+\cdots
$$

where $f^{i j}=\left(\gamma^{-1}\right)^{(0) i j}$ is the inverse of $f_{i j}$. It is easy to show that $\left(\gamma^{-1}\right)^{(n) i j}(n \geq 1)$ satisfies the following differential equation.

$$
\partial_{t}\left(\gamma^{-1}\right)^{(n) i j}=-2 \sum_{p=1}^{n} A_{k}^{(p) i}\left(\gamma^{-1}\right)^{(n-p) k j}
$$

There are some useful identities. The generalized Bianchi identity (14) leads to

$$
\partial_{j} Z_{i}^{(n) j}+3 \sum_{p=0}^{n}\left(Z_{i}^{(p) j}-\frac{1}{3} Z^{(p)} \delta_{i}^{j}\right) \partial_{j} \zeta^{(n-p)}-\frac{1}{2} \sum_{p=0}^{n} \sum_{q=0}^{n-p} Z^{(p) j}{ }_{l}\left(\gamma^{-1}\right)^{(q) l k} \partial_{i} \gamma_{j k}^{(n-p-q)}=0
$$


where $Z^{(n) i}{ }_{j}$ and $Z^{(n)}$ are the $n$-th order parts of $Z^{i}{ }_{j}$ and $Z$, respectively. The conditions $A^{i}{ }_{i}=0, \partial_{i} \ln \operatorname{det} \gamma=0$, $\gamma_{i j}-\gamma_{j i}=0, \gamma_{i k} A_{j}^{k}-\gamma_{j k} A_{i}^{k}=0$ and $A^{i}{ }_{j}-\gamma_{j k} A_{l}^{k}\left(\gamma^{-1}\right)^{l i}=0$ lead to the following identities.

$$
\begin{aligned}
& A_{i}^{(n) i}=0, \quad \sum_{p=0}^{n}\left(\gamma^{-1}\right)^{(p) j k} \partial_{i} \gamma_{j k}^{(n-p)}=0, \quad \gamma_{i j}^{(n)}-\gamma_{j i}^{(n)}=0, \\
& \sum_{p=0}^{n-1}\left(\gamma_{i k}^{(p)} A_{j}^{(n-p) k}-\gamma_{j k}^{(p)} A^{(n-p) k}{ }_{i}\right)=0, \quad A_{j}^{(n) i}-\sum_{p=0}^{n-1} \sum_{q=0}^{n-p-1} \gamma_{j k}^{(p)} A^{(n-p-q) k}{ }_{l}\left(\gamma^{-1}\right)^{(q) l i}=0 .
\end{aligned}
$$

\section{D. $O(\epsilon)$ solution}

For the first order $(n=1)$, eqs.(38.41) are reduced to

$$
\begin{aligned}
\partial_{t}\left(a^{3} K^{(1)}\right) & =0, \\
\partial_{t}\left(a^{3} A^{(1) i}\right) & =0, \\
\partial_{t} \zeta^{(1)} & =\frac{1}{3} K^{(1)}, \\
\partial_{t} \gamma_{i j}^{(1)} & =2 f_{i k} A_{j}^{(1) k} .
\end{aligned}
$$

Note that $Z_{j}^{(1) i}=0$. Integrating these equations, we obtain

$$
\begin{aligned}
K^{(1)} & =\frac{C^{(1)}}{a(t)^{3}}, \\
A_{j}^{(1) i} & =\frac{C^{(1) i}}{a(t)^{3}}, \\
\zeta^{(1)} & =\frac{C^{(1)}}{3} \int_{t_{i n}}^{t} \frac{d t^{\prime}}{a^{3}\left(t^{\prime}\right)}+\zeta_{i n}^{(1)}, \\
\gamma_{i j}^{(1)} & =2 f_{i k} C_{j}^{(1) k} \int_{t_{i n}}^{t} \frac{d t^{\prime}}{a^{3}\left(t^{\prime}\right)}+\gamma_{i n i j}^{(1)},
\end{aligned}
$$

where the integration constants $C^{(1)}, C_{j}^{(1) i}, \zeta_{i n}^{(1)}$ and $\gamma_{i n i j}^{(1)}$ depend on the spatial coordinates $\vec{x}^{i}$ only, and satisfy

$$
C_{i}^{(1) i}=0, \quad f_{i k} C_{j}^{(1) k}=f_{j k} C_{i}^{(1) k} .
$$

The two integration constants, $\zeta_{i n}^{(1)}$ and $\gamma_{i n i j}^{(1)}$, can be absorbed into the zero-th order counterparts, $\zeta_{i n}^{(0)}$ and $\gamma_{i n i j}^{(0)}$. Thus, without loss of generality, we can set

$$
\zeta_{i n}^{(1)}=0, \quad \gamma_{i n i j}^{(1)}=0 .
$$

Finally, the momentum constraint equation (42) with $n=1$ leads to the following relation among the remaining integration constants, $C^{(1)}, C_{j}^{(1) i}, \zeta^{(0)}$ and $f_{i j}$.

$$
\partial_{j} C^{(1) j}{ }_{i}+3 C^{(1) j}{ }_{i} \partial_{j} \zeta^{(0)}-\frac{1}{2} C^{(1) j}{ }_{l} f^{l k} \partial_{i} f_{j k}-\frac{1}{3}(3 \lambda-1) \partial_{i} C^{(1)}=0,
$$

where $f^{i j}$ is the inverse of $f_{i j}$. 


\section{E. $O\left(\epsilon^{2}\right)$ solution}

With the first order solution obtained in the previous subsection, we can solve the second order equations. In the second order, eqs.(38,41) become

$$
\begin{aligned}
a^{-3} \partial_{t}\left(a^{3} K^{(2)}\right) & =-\frac{1}{2}\left(K^{(1)}\right)^{2}-\frac{3}{2((3 \lambda-1))} A_{j}^{(1) i} A_{i}^{(1) j}-\frac{1}{2(3 \lambda-1)} a^{-2} \tilde{R} \\
a^{-3} \partial_{t}\left(a^{3} A_{j}^{(2) i}\right) & =-K^{(1)} A_{j}^{(1) i}-a^{-2}\left(\tilde{R}_{j}^{i}-\frac{1}{3} \tilde{R} \delta_{j}^{i}\right) \\
\partial_{t} \zeta^{(2)} & =\frac{1}{3} K^{(2)}, \\
\partial_{t} \gamma_{i j}^{(2)} & =2\left(f_{i k} A_{j}^{(2) k}+\gamma_{i k}^{(1)} A_{j}^{(1) k}\right),
\end{aligned}
$$

where $\tilde{R}_{j}^{i}$ and $\tilde{R}$ are Ricci tensor and Ricci scalar constructed from the 0-th order conformally-transformed metric $a^{-2} g_{i j}^{(0)}=e^{2 \zeta^{(0)}} f_{i j}$, and we have used the fact that $Z_{j}^{(2) i}=-a^{-2}\left(\tilde{R}_{j}^{i}-\frac{1}{2} \tilde{R} \delta^{i}{ }_{j}\right)$. By integrating these equations we obtain

$$
\begin{aligned}
K^{(2)} & =-\frac{1}{2 a^{3}(t)}\left\{\left[\left(C^{(1)}\right)^{2}+\frac{3}{3 \lambda-1} C_{j}^{(1) i} C_{i}^{(1) j}\right] \int_{t_{i n}}^{t} \frac{d t^{\prime}}{a^{3}\left(t^{\prime}\right)}+\frac{\tilde{R}}{3 \lambda-1} \int_{t_{i n}}^{t} a\left(t^{\prime}\right) d t^{\prime}\right\}, \\
A_{j}^{(2) i} & =-\frac{1}{a^{3}(t)}\left\{C^{(1)} C_{j}^{(1) i} \int_{t_{i n}}^{t} \frac{d t^{\prime}}{a^{3}\left(t^{\prime}\right)}+\left(\tilde{R}_{j}^{i}-\frac{1}{3} \tilde{R} \delta_{j}^{i}\right) \int_{t_{i n}}^{t} a\left(t^{\prime}\right) d t^{\prime}\right\}, \\
\zeta^{(2)} & =-\frac{1}{6}\left\{\left[\left(C^{(1)}\right)^{2}+\frac{3}{3 \lambda-1} C_{j}^{(1) i} C_{i}^{(1) j}{ }_{i}\right] \int_{t_{i n}}^{t} \frac{d t^{\prime}}{a^{3}\left(t^{\prime}\right)} \int_{t_{i n}}^{t^{\prime}} \frac{d t^{\prime \prime}}{a^{3}\left(t^{\prime \prime}\right)}+\frac{\tilde{R}}{3 \lambda-1} \int_{t_{i n}}^{t} \frac{d t^{\prime}}{a^{3}\left(t^{\prime}\right)} \int_{t_{i n}}^{t^{\prime}} a\left(t^{\prime \prime}\right) d t^{\prime \prime}\right\}, \\
\gamma_{i j}^{(2)} & =2 f_{i k}\left[\left(2 C_{l}^{(1) k} C_{j}^{(1) l}-C^{(1)} C_{j}^{(1) k}\right) \int_{t_{i n}}^{t} \frac{d t^{\prime}}{a^{3}\left(t^{\prime}\right)} \int_{t_{i n}}^{t^{\prime}} \frac{d t^{\prime \prime}}{a^{3}\left(t^{\prime \prime}\right)}-\left(\tilde{R}_{j}^{k}-\frac{1}{3} \tilde{R} \delta_{j}^{k}\right) \int_{t_{i n}}^{t} \frac{d t^{\prime}}{a^{3}\left(t^{\prime}\right)} \int_{t_{i n}}^{t^{\prime}} a\left(t^{\prime \prime}\right) d t^{\prime \prime}\right](65
\end{aligned}
$$

where we have set

$$
\left.K^{(2)}\right|_{t=t_{i n}}=\left.A_{j}^{(2) i}\right|_{t=t_{i n}}=\left.\zeta^{(2)}\right|_{t=t_{i n}}=\left.\gamma_{i j}^{(2)}\right|_{t=t_{i n}}=0
$$

by redefinition of $C^{(1)}, C_{j}^{(1) i}, \zeta^{(0)}$ and $f_{i j}$, respectively.

Provided that the redefined integration constants $\left(C^{(1)}, C_{j}^{(1) i}, \zeta^{(0)}, f_{i j}\right)$ satisfy (57) up to $O\left(\epsilon^{3}\right)$, one can show that the solution (62,65) automatically satisfies the third-order momentum constraint equation

$$
\begin{aligned}
& \partial_{j} A^{(2) j}{ }_{i}+3 A^{(1) j}{ }_{i} \partial_{j} \zeta^{(1)}+3 A^{(2) j}{ }_{i} \partial_{j} \zeta^{(0)} \\
& \quad-\frac{1}{2} A^{(1) j}{ }_{l} f^{l k} \partial_{i} \gamma_{j k}^{(1)}-\frac{1}{2} A^{(1) j}{ }_{l}\left(\gamma^{-1}\right)^{(1) l k} \partial_{i} f_{j k}-\frac{1}{2} A^{(2) j}{ }_{l} f^{l k} \partial_{i} f_{j k}-\frac{1}{3}(3 \lambda-1) \partial_{i} K^{(2)}=0 .
\end{aligned}
$$

The proof is given in Appendix $\mathrm{A}$.

\section{F. $O\left(\epsilon^{n}\right)$ solution $(n \geq 2)$}

For general $n(\geq 2)$, the solution to eqs. 38, 41) is

$$
\begin{aligned}
K^{(n)} & =\frac{1}{a^{3}(t)} \int_{t_{i n}}^{t} d t^{\prime} a^{3}\left(t^{\prime}\right)\left[-\frac{1}{2} \sum_{p=1}^{n-1} K^{(p)} K^{(n-p)}-\frac{3}{2(3 \lambda-1)} \sum_{p=1}^{n-1} A_{j}^{(p) i} A_{j}^{(n-p) j}{ }_{i}-\frac{Z^{(n)}}{3 \lambda-1}\right] \\
A_{j}^{(n) i} & =\frac{1}{a^{3}(t)} \int_{t_{i n}}^{t} d t^{\prime} a^{3}\left(t^{\prime}\right)\left[-\sum_{p=1}^{n-1} K^{(p)} A_{j}^{(n-p) i}+Z_{j}^{(n) i}-\frac{1}{3} Z^{(n)} \delta_{j}^{i}\right] \\
\zeta_{j}^{(n)} & =\frac{1}{3} \int_{t_{i n}}^{t} d t^{\prime} K^{(n)} \\
\gamma_{i j}^{(n)} & =2 \int_{t_{i n}}^{t} d t^{\prime} \sum_{p=0}^{n-1} \gamma_{i k}^{(p)} A_{j}^{(n-p) k},
\end{aligned}
$$


where we have set

$$
\left.K^{(n)}\right|_{t=t_{i n}}=\left.A_{j}^{(n) i}\right|_{t=t_{i n}}=\left.\zeta^{(n)}\right|_{t=t_{i n}}=\left.\gamma_{i j}^{(n)}\right|_{t=t_{i n}}=0
$$

by redefinition of $C^{(1)}, C_{j}^{(1) i}, \zeta^{(0)}$ and $f_{i j}$, respectively. Note that, in subsection IIE, we have already set

$$
\left.\zeta^{(1)}\right|_{t=t_{i n}}=\left.\gamma_{i j}^{(1)}\right|_{t=t_{i n}}=0
$$

by redefinition of $\zeta^{(0)}$ and $f_{i j}$, respectively.

The initial condition for $\gamma_{i j}^{(n)}(n \geq 1)$ implies that $\left.\gamma_{i j}\right|_{t=t_{i n}}=f_{i j}$, that $\left.\left(\gamma^{-1}\right)^{i j}\right|_{t=t_{i n}}=f^{i j}$ and that $\left.\left(\gamma^{-1}\right)^{(n)} i j\right|_{t=t_{i n}}=0(n \geq 1)$. Therefore, for $n \geq 1$, the solution to (44) is

$$
\left(\gamma^{-1}\right)^{(n) i j}=-2 \int_{t_{i n}}^{t} d t^{\prime} \sum_{p=1}^{n} A_{k}^{(p) i}\left(\gamma^{-1}\right)^{(n-p) k j} .
$$

As shown in Appendix A, the solution (68, 71) automatically satisfies the $(n+1)$-th order momentum constraint equation (42), provided that the redefined integration constants $\left(C^{(1)}, C_{j}^{(1) i}, \zeta^{(0)}, f_{i j}\right)$ satisfy (57) up to $O\left(\epsilon^{n+1}\right)$.

\section{G. Number of physical degrees of freedom}

Our solution involves functions $\zeta^{(0)}(\vec{x}), f_{i j}(\vec{x}), C^{(1)}(\vec{x})$ and $C^{(1) i}{ }_{j}(\vec{x})$ of spatial coordinates as integration 'constant'. They are subject to the constraint (57). Also, as stated just after (22), our gauge fixing condition (22) leaves timeindependent spatial diffeomorphism as residual gauge freedom. Therefore, the number of physical degrees of freedom included in each integration 'constant' is

$$
\begin{array}{rll}
\zeta^{(0)}(\vec{x}) & \cdots & 1 \text { scalar growing mode }=1 \text { component }, \\
f_{i j}(\vec{x}) & \cdots & 2 \text { tensor growing modes }=5 \text { components }-3 \text { gauge }, \\
C^{(0)}(\vec{x}) & \cdots & 1 \text { scalar decaying mode }=1 \text { component }, \\
C_{j}^{(1) i}(\vec{x}) & \cdots & 2 \text { tensor decaying modes }=5 \text { components }-3 \text { constraints } .
\end{array}
$$

This is consistent with the fact that the Hořava-Lifshitz gravity includes not only a tensor graviton (2 propagating degrees of freedom) but also a scalar graviton (1 propagating degree of freedom).

\section{CONSERVED CURVATURE PERTURBATION}

In this section we propose a definition of nonlinear curvature perturbation and show that it is conserved up to the first order in the gradient expansion. This statement holds for any values of $\lambda$. Because of the conservation, this quantity is expected to be useful for the analysis of superhorizon evolution of nonlinear perturbation.

\section{A. Definition}

In general relativity, it is known that the curvature perturbation in the uniform density slice conserves up to the first order in the gradient expansion [7]. Motivated by this fact, we define the quantity $\mathcal{R}$ in Hořava-Lifshitz gravity by

$$
\mathcal{R}(t, \vec{x}) \equiv \zeta(\tilde{t}, \vec{x})+\ln \left[\frac{a(\tilde{t})}{a(t)}\right]
$$

where $\tilde{t}(t, \vec{x})=t+O(\epsilon)$ is the solution to

$$
\rho_{d m}^{(0)}(\tilde{t})+\delta \rho_{d m}(\tilde{t}, \vec{x})=\rho_{d m}^{(0)}(t)
$$


and

$$
\rho_{d m}^{(0)}(t) \equiv 3 M_{P l}^{2} H^{2}-\frac{2 M_{P l}^{2}}{3 \lambda-1} \Lambda, \quad \delta \rho_{d m}(t, \vec{x}) \equiv \frac{M_{P l}^{2}}{2}\left[R+\frac{2}{3}\left(K^{2}-9 H^{2}\right)-A_{j}^{i} A_{i}^{j}\right] .
$$

In the following we shall show that $\mathcal{R}$ is indeed conserved up to the first order in the gradient expansion by explicitly calculating it.

\section{B. Concrete expression and conservation up to $O(\epsilon)$}

According to the gradient expansion, we expand $\delta \rho_{d m}, \tilde{t}$ and $\mathcal{R}$ as

$$
\delta \rho_{d m}=\sum_{n=1}^{\infty} \rho_{d m}^{(n)}, \quad \tilde{t}=t+\sum_{n=1}^{\infty} \tilde{t}^{(n)}, \quad \mathcal{R}=\sum_{n=0}^{\infty} \mathcal{R}^{(n)},
$$

where a quantity with the superscript $(n)$ is of order $O\left(\epsilon^{n}\right)$.

At the order $O\left(\epsilon^{0}\right)$, we obtain

$$
\mathcal{R}^{(0)}=\zeta^{(0)}(\vec{x})
$$

and this is constant in time.

At the order $O(\epsilon)$, we obtain

$$
\rho_{d m}^{(1)}=2 M_{P l}^{2} H K^{(1)}, \quad \tilde{t}^{(1)}=-\frac{\rho_{d m}^{(1)}}{\partial_{t} \rho^{(0)}}=-\frac{K^{(1)}}{3 \partial_{t} H}, \quad \mathcal{R}^{(1)}=\zeta^{(1)}+H \tilde{t}^{(1)}=\frac{C^{(1)}}{3}\left[\int_{t_{i n}}^{t} \frac{d t^{\prime}}{a^{3}\left(t^{\prime}\right)}-\frac{H}{a^{3} \partial_{t} H}\right] .
$$

Thus, the time derivative of $\mathcal{R}^{(1)}$ is shown to vanish as

$$
\partial_{t} \mathcal{R}^{(1)}=\frac{C^{(1)} H}{3 a^{3}\left(\partial_{t} H\right)^{2}}\left(\partial_{t}^{2} H+3 H \partial_{t} H\right)=0,
$$

where we have used the time derivative of (36) to show the last equality.

We therefore conclude that $\mathcal{R}$ defined in (76) is conserved up to the first order in the gradient expansion. This statement holds for any values of $\lambda$.

\section{SUMMARY AND DISCUSSION}

We have performed a fully nonlinear analysis of superhorizon perturbation in Hořava-Lifshitz gravity by using the gradient expansion technique. In $\S$ 【一 we have presented a concrete expression for the solution of gravity equations up to the second order in the gradient expansion. We have also proven that the solution can be extended to any order of the gradient expansion, by showing that the solution to the dynamical equation satisfies the constraint equation at each order.

Based on the result, in $\S \llbracket$ we have proposed a definition of nonlinear curvature perturbation $\mathcal{R}$ in Hořava-Lifshitz gravity and have shown that it is conserved up to the first order in the gradient expansion.

It is known that, in the limit $\lambda \rightarrow 1$, the scalar graviton gets strongly coupled and the usual metric perturbation breaks down in the scalar graviton sector [3]. Here, we stress that the breakdown of the perturbative expansion does not necessarily lead to a loss of predictability since all coefficients of infinite number of terms in the perturbative expansion can be written in terms of finite parameters in the action if the theory is renormalizable. Indeed, for spherically symmetric, static, vacuum configurations, one can perform fully nonlinear analysis to show that the limit $\lambda \rightarrow 1$ is continuous and the general relativity is recovered in the limit [2]. This result may be considered as an analogue of Vainshtein effect and suggests the possibility that the scalar graviton may safely be decoupled from the rest of the world, i.e. the tensor graviton and the matter sector, in the limit.

The result of the present paper is based on the fully nonlinear analysis and may be considered as yet another example of the analogue of Vainshtein effect. Up to any order of the gradient expansion, the equations of motion and their solutions are manifestly regular in the limit $\lambda \rightarrow 1$. The solutions reduce to those in general relativity coupled to dark matter in the limit at low energy. 
In the present paper we have concentrated on the pure gravity system in the projectable Hořava-Lifshitz theory. Because of the existence of the scalar graviton, this simple system is still rich enough as a testing ground for the analogue of Vainshtein effect. Indeed, the so called "dark matter as integration constant" [5] drives non-trivial cosmological dynamics in this system, and thus the nonlinear analysis presented in the present paper provides a convincing evidence for the analogue of Vainshtein effect. It is certainly interesting and important to extend the nonlinear analysis to more general situations with matter contents [10].

\section{Acknowledgments}

K.I. acknowledges supports by the Grant-in-Aid for Scientific Research (A) No. 21244033. Part of this work was done during S.M.'s participation in YITP molecule-type workshop (T-10-05): Cosmological Perturbation and Cosmic Microwave Background. He thanks YITP for stimulating atmosphere and warm hospitality. The work of S.M. is supported by Grant-in-Aid for Scientific Research 17740134, 19GS0219, 21111006, 21540278, and by World Premier International Research Center Initiative (WPI Initiative). The authors are also supported by Japan-Russia Research Cooperative Program.

Appendix A: $(n+1)$-th order momentum constraint $(n \geq 2)$

In this appendix, by induction we prove that the $n$-th order solution (68) satisfies the $(n+1)$-th order momentum constraint equation (42) for $n \geq 2$.

The basic logic of the proof is to rewrite the left hand side of the $(n+1)$-th order constraint (42) as a linear combination of lower order constraints by using the explicit solution (68) [71). For this purpose we shall use the generalized Bianchi identity (45) and other identities (46). We also use the following identity for functions $f(t)$ and $g(t)$ satisfying $a^{3}\left(t_{i n}\right) f\left(t_{i n}\right) g\left(t_{i n}\right)=0$ :

$$
f(t) g(t)=\frac{1}{a^{3}(t)} \int_{t_{i n}}^{t} d t^{\prime} a^{3}\left(t^{\prime}\right)\left[a\left(t^{\prime}\right)^{-3} \partial_{t^{\prime}}\left(a^{3}\left(t^{\prime}\right) f\left(t^{\prime}\right)\right) \cdot g\left(t^{\prime}\right)+f\left(t^{\prime}\right) \cdot \partial_{t^{\prime}} g\left(t^{\prime}\right)\right] .
$$

By applying the identity (A1) to $(f(t), g(t))=\left(A^{(p) j}{ }_{i}, \partial_{j} \zeta^{(n-p)}\right)$ and $(f(t), g(t))=\left(A^{(p) j}{ }_{l},\left(\gamma^{-1}\right)^{(q) l k} \partial_{i} \gamma_{j k}^{(n-p-q)}\right)$, the left hand side of the $(n+1)$-th order momentum constraint equation (42) is rewritten as

$$
\begin{aligned}
\mathcal{C}_{i}^{(n+1)} \equiv & \partial_{j} A^{(n) j}{ }_{i}+3 \sum_{p=1}^{n} A^{(p) j}{ }_{i} \partial_{j} \zeta^{(n-p)}-\frac{1}{2} \sum_{p=1}^{n} \sum_{q=0}^{n-p} A^{(p) j}{ }_{l}\left(\gamma^{-1}\right)^{(q) l k} \partial_{i} \gamma_{j k}^{(n-p-q)}-\frac{1}{3}(3 \lambda-1) \partial_{i} K^{(n)} \\
= & \partial_{j} A^{(n) j}{ }_{i}+\frac{1}{a^{3}(t)} \int_{t_{i n}}^{t} d t^{\prime} a^{3}\left(t^{\prime}\right)\left\{3 \sum_{p=1}^{n}\left[a^{-3} \partial_{t^{\prime}}\left(a^{3} A^{(p) j}{ }_{i}\right) \partial_{j} \zeta^{(n-p)}+A^{(p) j}{ }_{i} \partial_{j}\left(\partial_{t^{\prime}} \zeta^{(n-p)}\right)\right]\right. \\
& -\frac{1}{2} \sum_{p=1}^{n} \sum_{q=0}^{n-p}\left[a^{-3} \partial_{t^{\prime}}\left(a^{3} A^{(p) j}{ }_{l}\right)\left(\gamma^{-1}\right)^{(q) l k} \partial_{i} \gamma_{j k}^{(n-p-q)}+A^{(p) j}{ }_{l} \partial_{t^{\prime}}\left(\left(\gamma^{-1}\right)^{(q) l k}\right) \partial_{i} \gamma_{j k}^{(n-p-q)}\right. \\
& \left.\left.+A^{(p) j}{ }_{l}\left(\gamma^{-1}\right)^{(q) l k} \partial_{i}\left(\partial_{t^{\prime}} \gamma_{j k}^{(n-p-q)}\right)\right]\right\}-\frac{1}{3}(3 \lambda-1) \partial_{i} K^{(n)} .
\end{aligned}
$$


Using (68, 69), (40,41) and (44), this is further rewritten as

$$
\begin{aligned}
& \mathcal{C}_{i}^{(n+1)}=\frac{1}{a^{3}(t)} \int_{t_{i n}}^{t} d t^{\prime} a^{3}\left(t^{\prime}\right)\left\{\partial_{j}\left(-\sum_{p=1}^{n-1} K^{(p)} A^{(n-p) j}{ }_{i}\right)\right. \\
& +3\left[\sum_{p=2}^{n}\left(-\sum_{q=1}^{p-1} K^{(q)} A^{(p-q) j}{ }_{i}\right) \partial_{j} \zeta^{(n-p)}+\sum_{p=1}^{n-1} A^{(p) j}{ }_{i} \partial_{j}\left(\frac{1}{3} K^{(n-p)}\right)\right] \\
& -\frac{1}{2} \sum_{p=1}^{n} \sum_{q=0}^{n-p}\left[\left(-\sum_{r=1}^{p-1} K^{(r)} A^{(p-r) j}\right)\left(\gamma^{-1}\right)^{(q) l k} \partial_{i} \gamma_{j k}^{(n-p-q)}\right. \\
& +A_{l}^{(p) j}\left(-2 \sum_{r=1}^{q} A_{m}^{(r) l}\left(\gamma^{-1}\right)^{(q-r) m k}\right) \partial_{i} \gamma_{j k}^{(n-p-q)} \\
& \left.+A^{(p) j}{ }_{l}\left(\gamma^{-1}\right)^{(q) l k} \partial_{i}\left(2 \sum_{r=1}^{n-p-q-1} \gamma_{j m}^{(r)} A_{k}^{(n-p-q-r) m}\right)\right] \\
& -\frac{1}{6}(3 \lambda-1) \partial_{i}\left(-\sum_{p=1}^{n-1} K^{(p)} K^{(n-p)}\right)-\frac{1}{2} \partial_{i}\left(-\sum_{p=1}^{n-1} A_{k}^{(p) j} A_{j}^{(n-p) k}\right) \\
& \left.+\frac{1}{6} \sum_{p=1}^{n} Z^{(p)} \sum_{q=0}^{n-p}\left(\gamma^{-1}\right)^{(q) j k} \partial_{i} \gamma_{j k}^{(n-p-q)}\right\},
\end{aligned}
$$

where we have used the generalized Bianchi identity (45). By using the identities (46) we finally obtain

$$
\mathcal{C}_{i}^{(n+1)}=-\frac{1}{a^{3}(t)} \int_{t_{i n}}^{t} d t^{\prime} a^{3}\left(t^{\prime}\right) \sum_{p=1}^{n-1} K^{(n-p)} \mathcal{C}_{i}^{(p+1)}
$$

Since we already know that $\mathcal{C}_{i}^{(2)}=0$ under the condition (57), this is enough to prove $\mathcal{C}^{(n+1)}=0$ for $n \geq 2$.

[1] P. Horava, Phys. Rev. D 79, 084008 (2009) arXiv:0901.3775 [hep-th]].

P. Horava, Phys. Rev. Lett. 102, 161301 (2009) arXiv:0902.3657 [hep-th]].

P. Horava and C. M. Melby-Thompson, Phys. Rev. D 82, 064027 (2010) [arXiv:1007.2410 [hep-th]].

[2] S. Mukohyama, Class. Quant. Grav. 27, 223101 (2010) arXiv:1007.5199 [hep-th]].

[3] C. Charmousis, G. Niz, A. Padilla and P. M. Saffin, JHEP 0908, 070 (2009) arXiv:0905.2579 [hep-th]].

[4] K. Koyama and F. Arroja, JHEP 1003, 061 (2010) arXiv:0910.1998 [hep-th]].

[5] S. Mukohyama, Phys. Rev. D 80, 064005 (2009) arXiv:0905.3563 [hep-th]].

[6] S. Mukohyama, JCAP 0906, 001 (2009) arXiv:0904.2190 [hep-th]].

G. Calcagni, JHEP 0909, 112 (2009) arXiv:0904.0829 [hep-th]].

R. Brandenberger, Phys. Rev. D 80, 043516 (2009) arXiv:0904.2835 [hep-th]].

E. Kiritsis and G. Kofinas, Nucl. Phys. B 821, 467 (2009) arXiv:0904.1334 [hep-th]].

S. Maeda, S. Mukohyama and T. Shiromizu, Phys. Rev. D 80, 123538 (2009) arXiv:0909.2149 [astro-ph.CO]].

S. Mukohyama, K. Nakayama, F. Takahashi and S. Yokoyama, Phys. Lett. B 679, 6 (2009) arXiv:0905.0055 [hep-th]].

T. Takahashi and J. Soda, Phys. Rev. Lett. 102, 231301 (2009) arXiv:0904.0554 [hep-th]].

S. Mukohyama, JCAP 0909, 005 (2009) arXiv:0906.5069 [hep-th]].

M. Henneaux, A. Kleinschmidt and G. Lucena Gomez, Phys. Rev. D 81, 064002 (2010) arXiv:0912.0399 [hep-th]].

A. Wang and R. Maartens, Phys. Rev. D 81, 024009 (2010) arXiv:0907.1748 [hep-th]].

D. Blas, O. Pujolas and S. Sibiryakov, Phys. Rev. Lett. 104, 181302 (2010) arXiv:0909.3525 [hep-th]].

K. Izumi and S. Mukohyama, Phys. Rev. D 81, 044008 (2010) arXiv:0911.1814 [hep-th]].

K. Maeda, Y. Misonoh and T. Kobayashi, Phys. Rev. D 82, 064024 (2010) [arXiv:1006.2739 [hep-th]].

T. Kobayashi, Y. Urakawa and M. Yamaguchi, JCAP 0911, 015 (2009) arXiv:0908.1005 [astro-ph.CO]].

T. Harada, U. Miyamoto and N. Tsukamoto, Int. J. Mod. Phys. D 20, 111 (2011) arXiv:0911.1187 [gr-qc]].

K. Izumi, T. Kobayashi and S. Mukohyama, JCAP 1010, 031 (2010) arXiv:1008.1406 [hep-th]].

A. E. Gumrukcuoglu and S. Mukohyama, arXiv:1104.2087 [hep-th].

[7] D. S. Salopek and J. R. Bond, Phys. Rev. D 42, 3936 (1990).

D. H. Lyth, K. A. Malik and M. Sasaki, JCAP 0505, 004 (2005) arXiv:astro-ph/0411220. 
[8] A. A. Starobinsky, JETP Lett. 42, 152 (1985) [Pisma Zh. Eksp. Teor. Fiz. 42, 124 (1985)].

Y. Nambu and A. Taruya, Class. Quant. Grav. 13, 705 (1996) arXiv:astro-ph/9411013.

H. Kodama and T. Hamazaki, Phys. Rev. D 57, 7177 (1998) arXiv:gr-qc/9712045].

S. M. Leach, M. Sasaki, D. Wands and A. R. Liddle, Phys. Rev. D 64, 023512 (2001) arXiv:astro-ph/0101406.

O. Seto, J. Yokoyama and H. Kodama, Phys. Rev. D 61, 103504 (2000) arXiv:astro-ph/9911119.

R. K. Jain, P. Chingangbam and L. Sriramkumar, JCAP 0710, 003 (2007) arXiv:astro-ph/0703762].

R. K. Jain, P. Chingangbam, L. Sriramkumar and T. Souradeep, Phys. Rev. D 82, 023509 (2010) arXiv:0904.2518 [astroph.CO]].

Y. Tanaka and M. Sasaki, Prog. Theor. Phys. 117, 633 (2007) arXiv:gr-qc/0612191.

Y. Tanaka and M. Sasaki, Prog. Theor. Phys. 118, 455 (2007) arXiv:0706.0678 [gr-qc]].

Y. Takamizu and S. Mukohyama, JCAP 0901, 013 (2009) arXiv:0810.0746 [gr-qc]].

Y. Takamizu, S. Mukohyama, M. Sasaki and Y. Tanaka, 'JCAP 1006, 019 (2010) arXiv:1004.1870 [astro-ph.CO]].

[9] A. I. Vainshtein, Phys. Lett. B 39, 393 (1972).

[10] A. E. Gumrukcuoglu, S. Mukohyama and A. Wang, work in progress. 